\title{
Botulinum Toxin Type A Satisfaction in Different Neurological Disorders
}

\author{
Jasem Yousef Al-Hashel1,2*, Doaa Youssry Soliman1,3, \\ Ismail Ibrahim Ismail ${ }^{1}$, Samar Farouk Ahmed ${ }^{1,4}$ \\ ${ }^{1}$ Department of Neurology, Ibn Sina Hospital, Kuwait City, Kuwait \\ ${ }^{2}$ Department of Medicine, Faculty of Medicine, Health Sciences Centre, Kuwait University, Kuwait City, Kuwait \\ ${ }^{3}$ Department of Neurology, Cairo University, Cairo, Egypt \\ ${ }^{4}$ Department of Neuropsychiatry, Faculty of Medicine, Minia University, Minia, Egypt \\ Email: ^dralhashel@hotmail.com, ^jasemkumsa@hotmail.com,doaayoussry@gmail.com, \\ dr.ismail.ibrahim2012@gmail.com,samerelshayb@hotmail.com
}

How to cite this paper: Al-Hashel, J.Y. Soliman, D.Y., Ismail, I.I. and Ahmed, S.F. (2022) Botulinum Toxin Type A Satisfaction in Different Neurological Disorders. Neuroscience \& Medicine, 13, 1-16. https://doi.org/10.4236/nm.2022.131001

Received: December 18, 2021 Accepted: February 20, 2022 Published: February 23, 2022

Copyright $\odot 2022$ by author(s) and Scientific Research Publishing Inc. This work is licensed under the Creative Commons Attribution International License (CC BY 4.0). http://creativecommons.org/licenses/by/4.0/

\begin{abstract}
Background: The botulinum toxin type A (BoNT-A) is used in a wide range of neurological diseases. We aimed to study the overall patients/caregivers' satisfaction with BoNT-A treatment in different neurological conditions. Methods: Prospective study included patients who had received at least two BoNTA treatment sessions. They were asked to rate overall treatment satisfaction at the peak of treatment effect on a 1 to 10 scale $(1=$ not at all satisfied; $10=$ fully satisfied). Subjects with a rating of 1 to 3 were classified as not at all satisfied, those with a rating of 4 to7 as somewhat satisfied, and those with a rating of 8 to10 as very satisfied. Treatment satisfaction questionnaire for medicine (TSQM) was assessed at the end of observational period. Quality of life QOL was assessed before BoNT-A treatment and at the last visit. Results: The study was conducted from first April 2014 to August 2021. 548 patients were identified with mean age $43.66 \pm 14.50$. Most of participants 389 (71\%) were female. At the end of observational period, the mean satisfaction was $7.28 \pm$ 1.78 . There was a highly significant difference $(P<0.0001)$ for treatment satisfaction among different neurological disorders. Total TSQM with BoNT-A was the highest for axillary hyperhidrosis $(\mathrm{HH})(90.45 \pm 0.62)$, followed by chronic migraine $(72.13 \pm 1.59)$ and the least TSQM was reported in writer's cramp (40.44 \pm 1.57$)$. Overall satisfaction with BoNT-A at the peak of treatment effect among patients with axillary HH (100\%), palmar HH (94.4\%), neuropathic pain syndromes (85.7\%), planter $\mathrm{HH}(90 \%)$, trigeminal neuralgia $(80 \%)$. While patients with cervical dystonia (86.7\%) and musculoskeletal pain $(80 \%)$ patients were somewhat satisfied. Not at all satisfied rates were recorded among writer's cramp patients (40\%). Total TSQM with BoNT-A was highest for axillary HH $90.45 \pm 0.62$ and least satisfaction was reported in writer's
\end{abstract}


cramp $40.44 \pm 1.57$. QOL significantly improved at the end of observational period (71.93 versus 104.52; $P<0.0001$ ). We reported a positive significant correlation between BoNT-A treatment satisfaction and treatment adherence (r $=0.242, P=0.001$ ). Conclusion: Satisfaction with BoNT-A therapy for different neurological disorders is overall good. The highest patient satisfaction was observed with primary focal $\mathrm{HH}$, and the least satisfaction was observed in writer's cramp. BoNT-A therapy improved QOL.

\section{Keywords}

Botulinum Toxin Type A, Satisfaction, Headache, Dystonia, Movement Disorders, Hyperhidrosis

\section{Background}

Botulinum toxin is the exotoxin of a gram-positive bacterium called "Clostridium Botulinum" that blocks the release of acetylcholine into the neural junction and, leads to reduced activity of the muscles and glands [1]. There are seven antigenically distinct toxins (A, B, C, D, E, F, G) [2]. Among these, type A toxin is the most stable and commercially used for medical treatment [3]. Botulinum toxin type A (BoNT-A) is composed of a heavy chain, which is responsible for the specific binding on the cholinergic fibers to' endings, and a light chain which is responsible for its enzymatic activity (zinc-dependent endopeptidase) [4]. It causes presynaptic blockage of the release of acetylcholine at the neuromuscular junction, thereby causing temporary muscle weakness lasting for 3 to 4 months. It is used to treat clinical disorders characterized by muscle hyperactivity. It has been extensively used for the treatment of different neurological disorders and was found to be well tolerated and highly effective [5] [6].

The American Food and Drug Administration (FDA) approved botulinum toxin for the treatment of strabismus, blepharospasm, cervical dystonia, glabellar facial lines, axillary hyperhidrosis, chronic migraine, and lateral canthal lines [7] [8]. Also, BoNT-A has been introduced as a useful therapeutic agent for the management of masticatory myofascial pain due to hyperactivity and spasm of the muscles of mastication and spasticity of the upper and lower extremities [1]. BoNT-A was recommended for the treatment of upper limb spasticity whatever the cause [9]. Previous studies concluded that repeated injections of BoNT-A for the treatment of post-stroke upper-limb spasticity led to improvements in muscle tone it was well tolerated, with no serious adverse events [10].

It is being used off-label for chronic lower back pain, traumatic brain injury, cerebral palsy, achalasia, and bruxism [8].

Adherence to medications is linked to patient satisfaction and it has a major concerned in health care. Medication satisfaction has can be measured as an outcome measure [11].

There is a growing interest in patient satisfaction with their therapies. Patient 
satisfaction with treatment is a valuable measure of treatment efficacy moreover, treatment satisfaction is associated with better compliance and adherence. There is a need to evaluate and improve patients' treatment satisfaction [12] [13].

Satisfaction with BoNT-A treatment among patients with different neurological disorders was found to be variable. Patient satisfaction with medication can be influenced by several factors such as the efficacy of therapy, convenience, or side effects of the medicine. Satisfaction with BoNT-A therapy is associated with better adherence, and persistence to treatment [12]. Satisfaction with BoNT-A therapy also improves quality of life [6].

Many factors affect successful outcomes such as injector experience, patient feedback and satisfaction. It is important to collect information about the satisfaction with BoNT-A treatment because of its widespread use in the field of neurology. The purpose of our study is to characterize the overall patients/caregivers' satisfaction with BoNT-A treatment for symptom control in different neurological conditions at our clinic in the only tertiary hospital in Kuwait.

\section{Methods}

This is a prospective study which included patients with different neurological disorders. We reviewed outpatient clinic, Ibn Sina Hospital, Ministry of Health, Kuwait during first April 2014 till 31 August 2021. Subjects of both genders at different age groups who had received at least two BoNT-A treatment sessions were eligible for participation in the study. All patients who had a follow-up for at least one year were included whether they continued on BoNT-A treatment session or discontinued it to avoid any results bias. Patients who were treated with neuroleptics or other drugs that interfere with neuromuscular transmission, pregnant or lactating females, secondary disease in the patients with dystonia, patients with psychiatric disorders were excluded from the study. Investigations were requested as needed for each patient to confirm their diagnosis.

BoNT-A injection was performed by neurologists (JA, DY) who are experts in BoNT-A treatment. We used 100 IU BoNT-A (Botox ${ }^{\circledR}$, Allergan) diluted with 2 or $4 \mathrm{~mL}$ of sterile normal saline. The usual dilution of BoNT type A was 5 IU per $0.1 \mathrm{~mL}$. Each treatment cycle consisted of multiple injections into single or multiple muscles according to the neurological diagnosis. The injections in some applications were performed under electromyographic or stimulation ultrasonographic guidance whenever necessary. The dose for subsequent injections was modified according to the therapeutic response to the preceding injections. $\mathrm{Mi}$ graine patients were injected intramuscularly with Onabotulinumtoxin A according to the PREEMPT protocol, 155 units divided into 31 injection sites around the head and neck, with sessions occurring every 3 months [14]. BoNT-A injections are administered at baseline visit and each follow-up visit, reflects the usual clinical routine and standards of care.

Treating doctors offered the subjects to participate in the study. We recorded and analyzed data for subjects who agreed to participate in the study. Demo- 
graphic data, detailed medical history, physical and neurologic examination were recorded for each participant.

Satisfaction with BoNT-A treatment is assessed by the patient and/or caregiver. We asked them to rate overall treatment satisfaction at the peak of treatment effect on a numerical rating scale, visual analog scale (VAS) ranging from 1 to 10. The VAS score is a patient-reported measure of the current health state ranging from 0 (worst health imaginable) to 10 (best imaginable health state). 1 was defined as not at all satisfied and 10 as very satisfied. Also, subjects with a rating of 1 - 3 were classified as not at all satisfied, those with a rating of $4-7$ as somewhat satisfied, and those with a rating of $8-10$ as very satisfied [15]. Treatment Satisfaction Questionnaire for Medication (TSQM); version 1.4 and [16] the Quality of Life (QOL) [17] were recorded before initiation of treatment and the end of observational period.

The TSQM comprises fourteen items across four domains focusing on effectiveness (three items), side effects (five items), convenience (three items), and global satisfaction (three items) of the medication over the previous 2 - 3 weeks before last visit. It was proved to be a valid and reliable tool in several languages including Arabic.

Outcomes measured included change in QOL from baseline. Percentage of patients satisfied with BoNT-A treatment at the last visit was recorded and compared among different neurological disorders. The patients were interviewed twice; initially, prior to their first session treatment, and the second after their treatment. The changes in the answers were compared to determine satisfaction with BoNT-A treatment and its impact on quality of life. All items except item 4 have five or seven responses, scored from one (least satisfied) to five or seven (most satisfied). Item 4 has 2 responses (presence of side effects; yes or no). Item scores are summed to give four domain scores, which are in turn transformed to a scale of 0 - 100 [18].

This research is in accordance with ethical guidelines of the Council for International Organizations of Medical Sciences [19] and principles in the Declaration of Helsinki [20].

Statistical analysis was performed using Statistical Package for Social Sciences version 24.0. Descriptive statistics were used to summarize all data. Numerical variables were summarized by mean and standard deviation (SD). Categorical variables were summarized as counts and percentages. Categorical variables were compared using the Chi-square test, and continuous variables were compared using Student's t-test. One-Way ANOVA tests were used for intergroup comparisons. QoL scores before and after treatment were analyzed using paired $t$-tests. Pearson's correlations were performed for correlation between treatment satisfaction and disease characters. $P<0.05$ was considered statistically significant.

\section{Results}

A total of 548 patients were enrolled in this study, with mean age $43.66 \pm 14.50$. 
Most of the participants 389 (71\%) were female. At the end of observational period, the mean duration of neurological disorders was $10.61 \pm 6.92$ years and subjects had been receiving BoNT-A injections for a mean of $49.66 \pm 29.17$ months.

Table 1 displays demographic and disease characteristics of patients who received BoNT-A. Headache disorders were the most prevalent clinical disorder $240(43.7 \%)$ and the least frequent was neuropathic pain syndromes $7(1.3 \%)$. (Table 1).

Table 1. Deamographgic and characters of patients who received BoNT-A $(\mathrm{N}=548)$.

\begin{tabular}{|c|c|c|c|c|c|}
\hline Diagnosis & $\begin{array}{c}\text { Number of } \\
\text { patients (\%) }\end{array}$ & $\begin{array}{c}\text { Age } \\
M \pm S D\end{array}$ & $\begin{array}{c}\text { Female } \\
\text { Gender } \\
\mathrm{N}(\%)\end{array}$ & $\begin{array}{c}\text { Disease } \\
\text { duration in } \\
\text { years } \\
M \pm S D\end{array}$ & $\begin{array}{c}\text { Treatment } \\
\text { duration in } \\
\text { months } \\
M \pm S D\end{array}$ \\
\hline Headache disorders & $240(43.7)$ & & & & \\
\hline Chronic migraine & $213(38.9)$ & $45.15 \pm 11.47$ & $190(48.8)$ & $12.02 \pm 7.33$ & $30.18 \pm 2031$ \\
\hline Other headache disorders & $27(4.9 \%)$ & $44.37 \pm 11.15$ & $24(6.2)$ & $13.22 \pm 6.40$ & $28.00 \pm 19.45$ \\
\hline Hemifacial spasm and related disorders & $92(16.8)$ & & & & \\
\hline HFS & $73(13.3)$ & $52.24 \pm 13.24$ & $45(11.6)$ & $9.45 \pm 6.54$ & $35.34 \pm 33.73$ \\
\hline Blepharospasm & $14(2.6)$ & $50.92 \pm 14.16$ & $11(2.8)$ & $14.14 \pm 7.42$ & $74.36 \pm 40.39$ \\
\hline Eyelid apraxia & $5(0.9)$ & $27.25 \pm 14.93$ & $5(1.3)$ & $5.60 \pm 3.73$ & $47.20 \pm 38.48$ \\
\hline Primary focal hyperhidrosis & $74(13.5)$ & & & & \\
\hline Axillary & $46(8.4)$ & $33.85 \pm 12.63$ & $20(5.1)$ & $11.48 \pm 7.42$ & $43.89 \pm 31.74$ \\
\hline Palmar & $18(3.3)$ & $31.12 \pm 12.93$ & $7(1.8)$ & $10.67 \pm 6.85$ & $4.94 \pm 30.18$ \\
\hline Planter & $10(1.8)$ & $32.75 \pm 13.92$ & $5(1.3)$ & $12.80 \pm 7.61$ & $50.10 \pm 38.16$ \\
\hline Focal dystonia & $49(8.9)$ & & & & \\
\hline Oromandibular dystonia & $16(2.9)$ & $42.42 \pm 8.25$ & $10(2.6)$ & $4.94 \pm 4.15$ & $27.31 \pm 24.33$ \\
\hline Cervical dystonia & $15(2.7)$ & $42.86 \pm 12.90$ & $7(1.8)$ & $5.33 \pm 3.02$ & $33.33 \pm 20.17$ \\
\hline Other focal dystonia & $13(2.4)$ & $65.53 \pm 11.54$ & $8(2.1)$ & $16.62 \pm 5.90$ & $85.23 \pm 23.80$ \\
\hline Writer's cramp & $5(0.9)$ & $46.40 \pm 19.01$ & $3(0.8)$ & $5.60 \pm 2.71$ & $31.20 \pm 18.20$ \\
\hline Spasticity & $47(8.65)$ & & & & \\
\hline Adults & $29(5.3)$ & $43.21 \pm 17.18$ & $21(5.4)$ & $5.86 \pm 3.43$ & $40.07 \pm 28.76$ \\
\hline Children (CP) & $18(3.3)$ & $15.22 \pm 4.03$ & $9(2.3)$ & $7.5 \pm 4.32$ & $60.00 \pm 35.08$ \\
\hline Sialorrhea & $24(4.4)$ & $63.25 \pm 13.12$ & $13(3.3)$ & $10.29 \pm 4.11$ & $39.12 \pm 17.63$ \\
\hline Neuropathic pain disorders & $12(2.2)$ & & & & \\
\hline Trigeminal neuralgia & $5(0.9)$ & $30.00 \pm 7.97$ & $3(0.8)$ & $8.80 \pm 1.64$ & $27.20 \pm 18.36$ \\
\hline Other neuropathic pain disorders & $7(1.3)$ & $36.00 \pm 11.02$ & $3(0.8)$ & $13.00 \pm 4.62$ & $46.14 \pm 31.75$ \\
\hline Musculoskeletal pain & $10(1.8)$ & $50.30 \pm 7.17$ & $5(1.3)$ & $4.4 \pm 4.88$ & $27.80 \pm 30.93$ \\
\hline
\end{tabular}

BoNT/A = botulinum neurotoxin type A; SD = standard deviation; HFS: hemifacial spasm; CP: cerebral palsy; M: mean; SD: standard deviation. 
At the end of observational period, we recorded patient satisfaction with BoNT-A therapy for different neurological disorders at the peak of treatment effects. The Mean satisfaction assessed by VAS was $7.28 \pm 1.78$. Majority of patients $52.9 \%$ were very satisfied, while $42.2 \%$ were somewhat satisfied and $4.9 \%$ were not satisfied at all. Gender did not show significant difference for overall BoNT-A satisfaction $(P<0.08)$.

Overall satisfaction assessed by VAS; for axillary $(\mathrm{HH})(9.24 \pm 0.77)$, palmar $\mathrm{HH}(8.83 \pm 0.62)$, neuropathic pain syndromes $(8.57 \pm 0.79)$, blepharospasm $(8.50 \pm 1.22)$, planter $\mathrm{HH}(8.30 \pm 0.94)$, trigeminal neuralgia $(8.20 \pm 0.84)$, apraxia of eyelid opening $(7.60 \pm 1.14)$, hemifacial spasm (HFS) $(7.52 \pm 1.36)$, chronic migraine (7.23 \pm 1.69$)$, cerebral palsy $(7.06 \pm 1.66)$, musculoskeletal pain $(6.50 \pm 1.17)$, oromandibular dystonia $(6.43 \pm 1.99)$, other headache disorders $(6.33 \pm 1.17)$, sialorrhoea $(6.25 \pm 2.04)$, spasticity $(6.07 \pm 1.98)$, other focal dystonia $(6.23 \pm 1.53)$, cervical dystonia $(5.53 \pm 1.64)$, and writer's cramp $(4.40 \pm$ 1.67). There was a highly significant difference $(P<0.0001)$ for treatment satisfaction among different neurological disorders. Overall treatment satisfaction with BoNT-A was highest for axillary $\mathrm{HH}(9.20 \pm 0.86)$ and the least satisfaction was reported in writer's cramp $(4.40 \pm 1.67)$ (Table 2).

Overall satisfaction with BoNT-A at the peak of treatment effect was very satisfied among patients with axillary $\mathrm{HH}(100 \%)$, palmar $\mathrm{HH}(94.4 \%)$, other neuropathic pain syndromes (85.7\%), planter $\mathrm{HH}$ (90), trigeminal neuralgia (80). While somewhat satisfied at peak of treatment effect was more reported among patients with cervical dystonia (86.7\%) and musculoskeletal pain (80). Not at all satisfied rates were recorded among writer's cramp patients (40\%).

Table 3 displays total TSQM among different neurological disorders. Total TSQM with BoNT-A was highest for axillary $\mathrm{HH} 90.45 \pm 0.62$ and least satisfaction was reported in writer's cramp $40.44 \pm 1.57$.

Table 4 showed that QOL significantly improved among all patients at the end of the observational period, (71.93 versus 104.52; $P<0.0001)$.

Table 5 shows a negative significant correlation between BoNT treatment satisfaction and age of patients. The younger the age of the patients the more satisfaction with BoNT-A treatment $(\mathrm{r}=-0.099, P<0.022)$. We reported positive significant correlation between BoNT-A treatment satisfaction and disease duration $(\mathrm{r}=0.185, P<0.0001)$. The longer the disease duration, the more satisfaction to BoNT-A treatment. Adherence to treatment was significantly correlated with BoNT treatment satisfaction $(\mathrm{r}=0.242, P=0.001)$.

Table 2. BoNT-A satisfaction in different neurological disorders $(\mathrm{N}=548)$.

\begin{tabular}{lcccc}
\hline Diagnosis & $\begin{array}{c}\text { VAS Mean } \\
(\mathrm{SD})\end{array}$ & $\begin{array}{c}\text { Not Satisfied } \\
\mathbf{N}(\%)\end{array}$ & $\begin{array}{c}\text { Somewhat } \\
\text { Satisfied N (\%) }\end{array}$ & $\begin{array}{c}\text { Very Satisfied } \\
\text { N (\%) }\end{array}$ \\
\hline $\begin{array}{l}\text { Headache disorders } \\
\quad \text { Chronic migraine } \\
\text { Other headache disorders }\end{array}$ & $7.23 \pm 1.69$ & $11(5.2)$ & $91(42.7)$ & $111(52.1)$ \\
& $6.33 \pm 1.18$ & 0 & $21(77.8)$ & $6(22.2)$ \\
\hline
\end{tabular}




\section{Continued}

\section{Hemifacial spasm and related disorders}

HFS

$\begin{array}{cccc}7.52 \pm 1.36 & 0 & 35(47.9) & 38(52.1) \\ 8.50 \pm 1.22 & 0 & 3(21.4) & 11(78.6) \\ 7.60 \pm 1.14 & 2 & 2(40) & 3(60)\end{array}$

Blepharospasm

Eyelid apraxia

Primary focal hyperhidrosis

Axillary
Palmar
Planter

$\begin{array}{cccc}9.24 \pm 0.77 & 0 & 0 & 46(100) \\ 8.83 \pm 0.62 & 0 & 1(5.6) & 17(94.4) \\ 8.30 \pm 0.95 & 0 & 1(10) & 9(90)\end{array}$

\section{Focal dystonia}

Oromandibular dystonia

Cervical dystonia

Other focal dystonia

Writer's cramp

$\begin{array}{cccc}6.43 \pm 1.99 & 3(18.8) & 7(43.8) & 6(37.5) \\ 5.53 \pm 1.64 & 2(13.3) & 13(86.7) & 0 \\ 6.23 \pm 1.54 & 1(7.7) & 10(76.9) & 2(15.4) \\ 4.40 \pm 1.67 & 2(40) & 3(60) & 0\end{array}$

Spasticity

Adults

Children (CP)

\section{Sialorrhea}

\section{Neuropathic pain disorders}

Trigeminal neuralgia

Neuropathic pain disorders

Musculoskeletal pain

$P$ value

$6.07 \pm 1.98$

5 (17.2)

$13(44.8)$

$11(37.9)$

$7.17 \pm 1.66$

0

$8(44.4)$

$10(55.6)$

$6.25 \pm 2.04$

$3(12.5)$

$13(54.2)$

$8(33.3)$

$\begin{array}{cccc}8.20 \pm 0.83 & 0 & 1(40) & 4(80) \\ 8.57 \pm 0.79 & 0 & 1(14.3) & 6(85.7) \\ 6.50 \pm 1.18 & 0 & 8(80) & 2(20) \\ 0.001^{\star} & 0.001^{\star *} & 0.01^{\star *} & 0.001^{\star *}\end{array}$

BoNT/A = botulinum neurotoxin type A; SD = standard deviation; HFS: hemifacial spasm; CP: cerebral palsy; M: mean; SD: standard deviation; VAS = visual analogue scale; ${ }^{\star}=$ significant, one way ANOVA; ** = significant Chi-square test.

Table 3. TSQM among patients with different neurological disorders on BoNT-A therapy $(\mathrm{N}=548)$.

Diagnosis

\section{Headache disorders}

Chronic migraine

Other headache disorders

\section{Hemifacial spasm and related disorders}

HFS

Blepharospasm

Eyelid apraxia

Primary focal hyperhidrosis

Axillary
Palmar
Planter
$70.42 \pm 1.16$

Total TSQM Mean (SD)

$72.13 \pm 1.59$

$63.23 \pm 1.11$

$82.30 \pm 1.10$

$71.30 \pm 1.15$

$90.45 \pm 0.62$

$81.13 \pm 0.64$

$80.14 \pm 1.05$ 


\section{Continued}

Focal dystonia

Oromandibular dystonia

$61.56 \pm 2.09$

Cervical dystonia

$50.52 \pm 1.74$

Other focal dystonia

$63.02 \pm 1.34$

Writer's cramp

$40.44 \pm 1.57$

Spasticity

Adults

$62.16 \pm 2.08$

Children (CP)

$72.26 \pm 1.86$

Sialorrhea

$62.15 \pm 1.94$

\section{Neuropathic pain disorders}

Trigeminal neuralgia

$81.29 \pm 1.03$

Neuropathic pain disorders

$80.58 \pm 1.09$

Musculoskeletal pain

$61.53 \pm 1.34$

$P$ value

$0.001^{\star}$

BoNT/A = botulinum neurotoxin type A; SD = standard deviation; HFS: hemifacial spasm; CP: cerebral palsy; M: mean; SD: standard deviation; TSQM = Treatment Satisfaction Questionnaire for Medication; ${ }^{*}=$ significant, paired $t$-tests.

Table 4. Change of QOL among patients with different neurological disorders on BoNTA therapy $(\mathrm{N}=548)$.

\begin{tabular}{|c|c|c|c|}
\hline Diagnosis & $\begin{array}{l}\text { QOL before } \\
\text { treatment } \\
\text { Mean (SD) }\end{array}$ & $\begin{array}{l}\text { QOL after } \\
\text { treatment } \\
\text { Mean (SD) }\end{array}$ & $\mathbf{P}$ \\
\hline \multicolumn{4}{|l|}{ Headache disorders } \\
\hline Chronic migraine & $80.12 \pm 1.30$ & $112.15 \pm 2.71$ & $0.001^{*}$ \\
\hline Other headache disorders & $73.21 \pm 2.10$ & $101.03 \pm 1.10$ & \\
\hline \multicolumn{4}{|c|}{ Hemifacial spasm and related disorders } \\
\hline HFS & $60.12 \pm 1.13$ & $90.12 \pm 1.13$ & $0.01^{\star}$ \\
\hline Blepharospasm & $72.10 \pm 2.11$ & $98.30 \pm 2.10$ & $0.03^{\star}$ \\
\hline Eyelid apraxia & $69.10 \pm 1.19$ & $96.10 \pm 1.12$ & $0.04^{*}$ \\
\hline \multicolumn{4}{|l|}{ Primary focal hyperhidrosis } \\
\hline Axillary & $60.15 \pm 1.63$ & $90.35 \pm 1.02$ & $0.002^{*}$ \\
\hline Palmar & $72.15 \pm 0.62$ & $101.11 \pm 1.62$ & $0.0001^{\star}$ \\
\hline Planter & $70.12 \pm 1.07$ & $111.13 \pm 1.60$ & $0.0001^{*}$ \\
\hline \multicolumn{4}{|l|}{ Focal dystonia } \\
\hline Oromandibular dystonia & $65.16 \pm 1.09$ & $90.16 \pm 1.01$ & $0.03^{*}$ \\
\hline Cervical dystonia & $58.51 \pm 1.73$ & $80.12 \pm 1.72$ & $0.004^{*}$ \\
\hline Other focal dystonia & $68.12 \pm 1.30$ & $83.42 \pm 0.89$ & $0.03^{*}$ \\
\hline Writer's cramp & $61.41 \pm 1.52$ & $65.30 \pm 1.51$ & 0.610 \\
\hline
\end{tabular}




\section{Continued}

\section{Spasticity}

Adults

Children (CP)

$82.15 \pm 2.18$

$99.11 \pm 1.18$

$0.050^{*}$

Sialorrhea

$92.13 \pm 1.80$

$108.16 \pm 1.06$

$0.01^{*}$

$82.20 \pm 2.03$

$102.11 \pm 1.13$

$0.001^{*}$

\section{Neuropathic pain disorders}

Trigeminal neuralgia

$80.09 \pm 1.02$

$99.39 \pm 1.56$

$0.02^{*}$

Neuropathic pain disorders

$90.18 \pm 1.39$

$112.18 \pm 1.20$

$0.01^{*}$

Musculoskeletal pain

$71.52 \pm 1.31$

$91.23 \pm 1.04$

$0.03^{*}$

BoNT/A = botulinum neurotoxin type A; SD = standard deviation; HFS: hemifacial spasm; $\mathrm{CP}$ : cerebral palsy; M: mean; SD: standard deviation; $\mathrm{QOL}=$ Quality of Life; ${ }^{\star}=$ significant, one way ANOVA.

Table 5. Correlation between satisfaction and disease characters.

\begin{tabular}{ccccc}
\hline Variables & Age & Gender & $\begin{array}{c}\text { Disease } \\
\text { duration }\end{array}$ & $\begin{array}{c}\text { Treatment } \\
\text { adherence }\end{array}$ \\
\hline Satisfaction for treatment & $\mathrm{R}=-0.099$ & $\mathrm{R}=0.018$ & $\mathrm{R}=0.185$ & $\mathrm{R}=0.242$ \\
& $P<0.022^{\star}$ & $P<0.674$ & $P<0.0001^{\star}$ & $P<0.001^{\star}$ \\
\hline
\end{tabular}

${ }^{*}=$ Significant.

\section{Discussion}

To date, most studies on satisfaction with BoNT-A in neurological disorders have been either small or very focused on specific disorders. Here we present a tertiary center experience with a larger number of patients with several neurological disorders. Patient satisfaction generally followed the onset, peak, and trough of efficacy. However, treatment outcome is individual and time to onset, peak, and trough of efficacy varies between patients [21]. This study was conducted to determine the level of overall satisfaction with BoNT-A treatment at the time of peak effect for different neurological disorders.

Our cohort included 548 subjects. Most of them were women with a mean age of 43.66 years.

Most of our patients were generally satisfied with their therapy at the time of peak of the effect, $52.9 \%$ were very satisfied; $42.2 \%$ of patients were somewhat satisfied and only $4.9 \%$ were not at all satisfied.

This study showed that all patient groups showed satisfaction with their BoNT-A treatment. Highest patient satisfaction was observed with primary focal $\mathrm{HH}$, and the least satisfaction was observed in focal dystonia which is cervical dystonia and writer's cramp. The satisfaction level to BoNT-A treatment among different diagnoses could be explained by different injection protocols for the different diagnoses as a site of injection, difficulty in reaching the target muscle, the correct dosing and injection interval or higher expectation by patients or their caregivers exceeding what is reasonably possible. 
The highest TSQM among our cohort was seen in HH, either axillary, palmar, or planter. This is in agreement with previously published studies. Similar to other published data that recorded $50 \%$ of focal $\mathrm{HH}$ patients reported satisfaction within the first week of treatment and increased to $94 \%$ after the second week [22] [23]. Hyperhidrosis is a problem with significant embarrassment. Treating such problems with BOT-A is easy and the treatment effect can last more than six months in most patients, likely this explains their high satisfaction.

Lowest patient TSQM for BoNT-A in our study was seen among cervical dystonia patients and writer's cramp patients. This could be explained by several factors other than symptom control as nonmotor symptoms or unrealistic expectations [13]. Cervical dystonia and writer's cramp both are focal dystonia. Both of them are difficult to inject and reaching the culprit muscle is not easy and affected patients may need several attempts to reach a good result despite using electromyography or ultrasonography. The mean TSQM for oromandibular dystonia patients was 61.6 which is consistent with a previous study of Meral who reported a mean satisfaction score of 6.74/10, six weeks after injection [24].

Chronic migraine was the most common enrolled in our cohort. A possible reason is the high prevalence of primary headache, migraine and chronic headache $61 \%, 23 \%$ and $5.6 \%$ respectively in Kuwait [25]. TSQM for BoNT-A in chronic migraine patients in our cohort was $(72.13 \pm 1.59)$ which was better than other headache disorders $(63.23 \pm 1.11)$ including cluster and tension-type headaches. Our results are in line with a previous study that reported $85 \%$ of chronic migraine patients showed over all good satisfaction at the end of observational period [26] [27]. Similarly, Cady et al. assessed treatment satisfaction using Migraine Impact Questionnaire (MIQ) and found that BoNT-A treated subjects showed improvement in 11 of 13 and 7 of 13 points at months 3 and 6, respectively compared to no improvement in the placebo group [28].

Our study also showed TSQM for BoNT-A in other painful conditions as Trigeminal neuralgia $(81.22 \pm 1.03)$ and other neuropathic pain $(80.58 \pm 1.09)$ similar to previous studies [29].

There is much basic scientific evidence for an analgesic effect of BoNT-A. Previous clinical trials confirmed its efficacy, safety and tolerability as prophylactic treatment of chronic migraine and also trigeminal neuralgia [30]. BoNT-A is effective at reducing pain in a number of disease states, including chronic migraine, cervical dystonia, neuropathic pain, lower back pain, spasticity, myofascial pain and bladder pain [31]. Our patients with trigeminal neuralgia showed good TSQM in $80 \%$ which was in line with a study by Li et al. who reported satisfaction in more than $90 \%$ of their cohort regarding improvement in the quality of life, emotional function and side effect burden [32]. BoNT-A inhibits neurogenic inflammation and peripheral sensitization, which potentially blocks the development of central sensitization [27]. Also, BoNT-A blocks the release of calcitonin gene-related peptide (CGRP) from the trigeminal neurons and improves migraine which could be another explanation [31]. 
The second frequent presentation in our cohort was Hemifacial spasm and related disorder $16.8 \%$. BoNT type A plays an important role in the treatment of HFS because the recommended pharmacological drugs usually have poor, brief, or no effect, and frequent adverse effects. Therefore, BoNT-A should be considered as the first-line therapy in patients with HFS [32]. Blepharospasm (BSP) patients were highly satisfied in our cohort with a TSQM score of $(80.50 \pm 1.22)$ which is in agreement with previous results [33].

In our study, a minority of the patients had spasticity, cerebral palsy and sialorrhea, these disorders had modest satisfactory results. Cerebral palsy is also mainly seen by pediatricians and is usually treated at their center.

Treatment with BoNT-A injections is well tolerated and may improve quality of life in different neurological conditions [15]. Our results support that QoL is significantly improved following BoNT-A treatment. Measurement of QoL can be used as an outcome measure following BoNT-A treatment. Most of our cohort were headache patients and they reported improvement of QOL after BoNT-A therapy. BoNT-A injections are effective therapy for chronic migraines and it improves patient quality of life [26].

Also, our results showed a significant correlation between BoNT treatment satisfaction and disease duration. This encourages neurologist to think of this option of treatment for patients who failed other treatment modalities. Most of our patients were regular on their visits to BoNT-A injection clinic. We reported significant positive correlation between treatment adherence and satisfaction to treatment. This is explained by the association between patients' satisfaction with treatment adherence and compliance. Improving components of treatment satisfaction, such as treatment convenience or side effects improves compliance and adherence [12].

Patient satisfaction has an impact on patients' health-related decisions and treatment-related behaviors, which affect the success of treatment outcomes. Patients' satisfaction with the services they receive improved treatment success, medical compliance, follow-through with treatment plans, and appropriate use of services [34].

Treatment satisfaction is an indicator of quality of health care system. Different factors influence treatment satisfaction as participant expectations, treatment procedure and treatment outcome, it remains unclear which factors contribute to satisfaction with each process and outcome attribute [35].

To improve patients'/caregivers' treatment satisfaction and optimize treatment outcomes, individualization of the injection protocol (site of injection, dosing and injection interval) should be considered. Ideally, patients should experience only a mild reappearance of symptoms towards the end of their individualized treatment cycle. A good understanding of the various factors associated with treatment satisfaction is vital when discussing the goals of treatment with patients and for planning treatment regimens. We should discuss realistic expectations with our patients. Since definitely unrealistic high expectation usually will end up with less or even no satisfaction period. 
Future research on satisfaction should explore the contribution of BoNT-A protocol and outcome factors on satisfaction to improve understanding of treatment attributes viewed favorably. This understanding optimizes treatment effectiveness.

\section{Conclusion}

To our knowledge, this study is the only study investigating patients'/caregivers' treatment satisfaction with BoNT-A treatment for different neurological indications to date. This study indicates that overall patient satisfaction with BoNT-A injections is good. The highest patient satisfaction is observed with primary focal $\mathrm{HH}$, and the least satisfaction with writer's cramp. Treatment satisfaction improves adherence to treatment. We reported a good overall satisfaction across different neurological conditions which was greater among those who were more adherent. This study also provides useful insight into the real-world use and treatment satisfaction with BoNT-A in different neurologic conditions.

\section{Strength and Limitations}

Several limitations of the present study should be considered. These include the small number of patients in some neurologic conditions, the satisfaction recorded at one point of treatment cycle (peak of the response). Patients' responses were based on subjective recollections. Other limitations are high heterogeneity of conditions, lack of other patient-oriented outcomes, lack of clinicians' and relatives' perspectives. The lack of routine safety assessment is another limitation. We didn't mention the full clinical and injection details because this is beyond the aim of our study. One of the limitations of our study is that we did not compare the satisfaction of botulinum toxin A treatment to other potential treatments. This study did not include a control group (without treatment or an alternative treatment). We did not study caregivers' and clinicians' perspectives (e.g. by applying the clinical global impression) and the importance of patient-oriented outcomes measures (e.g. goal-attainment scale). Despite these limitations, we think that the strength of our study consists of a heterogeneous patients' group and our results may contribute to the literature in the field of botulinum toxin usages and also patients' satisfaction with different modalities of treatment. Our study is a prospective design which included large datasets (almost 600 patients) for almost seven years.

\section{Ethics}

Study protocol and informed consent were reviewed and approved by Ibn Sina Medical Research Ethics Committee and the Institutional Review Board Committee of Ministry of health of the state of Kuwait. Written Informed consent was obtained from each subject prior to enrollment in the study. The study was conducted in accordance with ethical guidelines of the Council for International Organizations of Medical Sciences and principles in the Declaration of Helsinki. 


\section{Availability of Data}

Data are available at administrative section, neurology department, Ibn Sina hospital, Kuwait.

\section{Authors' Contributions}

JA designed the study, criticized and reviewed the manuscript. DY, SFA and III performed data collection and drafted the manuscript. SFA performed statistical analysis, drafted, criticized and reviewed the manuscript. JA and DY injected the patients with BoNT-A. All authors read and approved the final manuscript.

\section{Acknowledgments}

The authors would like to thank all patients who participated in the study. Our appreciation to nurse Enayaat Khairy at outpatient clinic in Ibn Sina Hospital for actively supporting the study. We would like to thank the the administrative department in Ibn Sina Hospital for actively supporting in the study.

\section{Conflicts of Interest}

The authors declare no conflicts of interest regarding the publication of this paper.

\section{References}

[1] Mor, N., Tang, C. and Blitzer, A. (2015) Temporomandibular Myofacial Pain Treated with Botulinum Toxin Injection. Toxins, 7, 2791-2800. https://doi.org/10.3390/toxins7082791

[2] Rossetto, O., Pirazzini, M. and Montecucco, C. (2014) Botulinum Neurotoxins: Genetic, Structural and Mechanistic Insights. Nature Reviews Microbiology, 12, 535-549. https://doi.org/10.1038/nrmicro3295

[3] Waller, R.R., Kennedy, R.H., Henderson, J.W. and Kesty, K.R. (1985) Management of Blepharospasm. Transactions of the American Ophthalmological Society, 83, 367-386.

[4] Ramachandran, R. and Yaksh, T.L. (2014) Therapeutic Use of Botulinum Toxin in Migraine: Mechanisms of Action. British Journal of Pharmacology, 171, 4177-4192. https://doi.org/10.1111/bph.12763

[5] Ward, A.B., Molenaers, G., Colosimo, C. and Berardelli, A. (2006) Clinical Value of Botulinum Toxin in Neurological Indications. European Journal of Neurology, 13, 20-26. https://doi.org/10.1111/j.1468-1331.2006.01650.x

[6] Amorelli, G., Spitz, M., Pereira, L.R. and Vasconcellos, L.F. (2017) Quality of Life and Epidemiological Profile of Patients Undergoing Botulinum Toxin Treatment. Revista Brasileira de Neurologia, 53, 23-26.

[7] Ihde, S.K. and Konstantinovic, V.S. (2007) The Therapeutic Use of Botulinum Toxin in Cervical and Maxillofacial Conditions: An Evidence-Based Review. Oral Surgery, Oral Medicine, Oral Pathology, Oral Radiology, and Endodontology, 104, e1-e11. https://doi.org/10.1016/j.tripleo.2007.02.004

[8] Tinastepe, N., Küçük, B.B. and Oral, K. (2015) Botulinum Toxin for the Treatment of Bruxism. CRANIO ${ }^{\oplus}$, 33, 292-298. https://doi.org/10.1179/2151090314Y.0000000022 
[9] Esquenazi, A., Albanese, A., Chancellor, M.B., Elovic, E., Segal, K.R., Simpson, D.M., Smith, C.P. and Ward, A.B. (2013) Evidence-Based Review and Assessment of Botulinum Neurotoxin for the Treatment of Adult Spasticity in the Upper Motor Neuron Syndrome. Toxicon, 67, 115-128. https://doi.org/10.1016/j.toxicon.2012.11.025

[10] Marciniak, C., Munin, M.C., Brashear, A., Rubin, B.S., Patel, A.T., Slawek, J., Hanschmann, A., Hiersemenzel, R. and Elovic, E.P. (2019) Incobotulinumtoxina Efficacy and Safety in Adults with Upper-Limb Spasticity Following Stroke: Results from the Open-Label Extension Period of a Phase 3 Study. Advances in therapy, 36, 187-199. https://doi.org/10.1007/s12325-018-0833-7

[11] Shikiar, R. and Rentz, A.M. (2004) Satisfaction with Medication: An Overview of Conceptual, Methodologic, and Regulatory Issues. Value in Health, 7, 204-215. https://doi.org/10.1111/j.1524-4733.2004.72252.x

[12] Barbosa, C.D., Balp, M.M., Kulich, K., Germain, N. and Rofail, D. (2012) A Literature Review to Explore the Link between Treatment Satisfaction and Adherence, Compliance, and Persistence. Patient Prefer Adherence, 6, 39-48.

https://doi.org/10.2147/PPA.S24752

[13] Colosimo, C., Charles, D., Misra, V.P., Maisonobe, P. and Om, S. (2019) INTEREST IN CD2 Study Group. How Satisfied Are Cervical Dystonia Patients after 3 Years of Botulinum Toxin Type A Treatment? Results from a Prospective, Long-Term Observational Study. Journal of Neurology, 266, 3038-3046.

https://doi.org/10.1007/s00415-019-09527-2

[14] Dodick, D.W., Turkel, C.C., DeGryse, R.E., Aurora, S.K., Silberstein, S.D., Lipton, R.B., et al. (2010) OnabotulinumtoxinA for Treatment of Chronic Migraine: Pooled Results from the Double-Blind, Randomized, Placebo-Controlled Phases of the PREEMPT Clinical Program. Headache: The Journal of Head and Face Pain, 50, 921-936. https://doi.org/10.1111/j.1526-4610.2010.01678.x

[15] Fezza, J, Burns, J., Woodward, J., Truong, D., Hedges, T. and Verma, A. (2016) A Cross-Sectional Structured Survey of Patients Receiving Botulinum Toxin Type A Treatment for Blepharospasm. Journal of the Neurological Sciences, 367, 56-62. https://doi.org/10.1016/j.jns.2016.05.033

[16] Vermersch, P., Hobart, J., Dive-Pouletty, C., Bozzi, S., Hass, S. and Coyle, P.K. (2017) Measuring Treatment Satisfaction in MS: Is the Treatment Satisfaction Questionnaire for Medication fit for purpose? Multiple Sclerosis Journal, 23, 604-613. https://doi.org/10.1177/1352458516657441

[17] The Whoqol Group (1998) The World Health Organization Quality of Life Assessment (WHOQOL): Development and Psychometric Properties. Social Science \& Medicine, 46, 1569-1585. https://doi.org/10.1016/S0277-9536(98)00009-4

[18] Atkinson, M.J., Sinha, A., Hass, S.L., Colman, S.S., Kumar, R.N., Brod, M. and Rowland, C.R. (2004) Validation of a General Measure of Treatment Satisfaction, the Treatment Satisfaction Questionnaire for Medication (TSQM), Using a National Panel Study of Chronic Disease. Health and Quality of Life Outcomes, 2, Article No. 12. https://doi.org/10.1186/1477-7525-2-12

[19] Foster, C.G. (1994) International Ethical Guidelines for Biomedical Research Involving Human Subjects. Journal of Medical Ethics, 20, 123-124.

https://doi.org/10.1136/jme.20.2.123

[20] World Medical Association (WMA) (2001) WMA Declaration of Helsinki-Ethical Principles for Medical Research Involving Human Subjects. JAMA, 310, 2191-2194. https://jamanetwork.com/journals/jama/fullarticle/1760318 
[21] Bensmail, D., Hanschmann, A. and Wissel, J. (2014) Satisfaction with Botulinum Toxin Treatment in Post-Stroke Spasticity: Results from Two Cross-Sectional Surveys (Patients and Physicians). Journal of Medical Economics, 17, 618-625. https://doi.org/10.3111/13696998.2014.925462

[22] Reis, G.M.D., Guerra, A.C.S. and Ferreira, J.P.A. (2011) Study of Patients with Hyperhidrosis Treated with Botulinum Toxin: A 10-Year Retrospective Analysis. Brazilian Journal of Plastic Surgery, 26, 582-590.

[23] Naumann, M., Lowe, N.J., Kumar, C.R. and Hamm, H. (2003) Botulinum Toxin Type A Is a Safe and Effective Treatment for Axillary Hyperhidrosis Over 16 Months: A Prospective Study. Archives of Dermatological, 139, 731-736. https://doi.org/10.1001/archderm.139.6.731

[24] Meral, S.E., Tüz, H.H. and Başlarll, Ö. (2019) Evaluation of Patient Satisfaction after Botulinum Toxin A Injection for the Management of Masticatory Myofascial Pain and Dysfunction-A Pilot Study. CRANIO ${ }^{\oplus}, 39,12-16$. https://doi.org/10.1080/08869634.2018.1562660

[25] Al-Hashel, J.Y., Ahmed, S.F. and Alroughani, R. (2017) Prevalence of Primary Headache Disorders in Kuwait. Neuroepidemiology, 48, 138-146. https://doi.org/10.1159/000478892

[26] Bruloy, E., Sinna, R., Grolleau, J.L., Bout-Roumazeilles, A., Berard, E. and Chaput, B. (2019) Botulinum Toxin versus Placebo: A Meta-Analysis of Prophylactic Treatment for Migraine. Plastic and Reconstructive Surgery, 143, 239-250. https://doi.org/10.1097/PRS.0000000000005111

[27] Schaefer, S.M., Gottschalk, C.H. and Jabbari, B. (2015) Treatment of Chronic Migraine with Focus on Botulinum Neurotoxins. Toxins, 7, 2615-2628.

https://doi.org/10.3390/toxins7072615

[28] Cady, R. and Schreiber, C. (2008) Botulinum Toxin Type A as Migraine Preventive Treatment in Patients Previously Failing Oral Prophylactic Treatment Due to Compliance Issues. Headache, 48, 900-913. https://doi.org/10.1111/j.1526-4610.2007.00953.x

[29] Luvisetto, S., Gazerani, P., Cianchetti C and Pavone F. (2015) Botulinum Toxin Type a as a Therapeutic Agent against Headache and Related Disorders. Toxins, 7, 3818-3844. https://doi.org/10.3390/toxins7093818

[30] Li, S., Lian, Y.J., Chen, Y., Zhang, H.-F., Ma, Y.-Q., He, C.-H., et al. (2014) Therapeutic Effect of Botulinum Toxin-A in 88 Patients with Trigeminal Neuralgia with 14-Month Follow-Up. The Journal of Headache and Pain, 15, Article No, 43. https://doi.org/10.1186/1129-2377-15-43

[31] Durham, P.L., Cady, R. and Cady, R. (2004) Regulation of Calcitonin Gene-Related Peptide Secretion from Trigeminal Nerve Cells by Botulinum Toxin Type A: Implications for Migraine Therapy. Headache: The Journal of Head and Face Pain, 44, 35-43. https://doi.org/10.1111/j.1526-4610.2004.04007.x

[32] Kenney, C. and Jankovic, J. (2008) Botulinum Toxin in the Treatment of Blepharospasm and Hemifacial Spasm. Journal of Neural Transmission, 115, 585-591. https://doi.org/10.1007/s00702-007-0768-7

[33] Burns, J., Woodward, J., Truong, D., Hedges, T. and Verma, A. (2016) A CrossSectional Structured Survey of Patients Receiving Botulinum Toxin Type A Treatment for Blepharospasm. Journal of the Neurological Sciences, 367, 56-62. https://doi.org/10.1016/j.jns.2016.05.033

[34] Atkinson, M.J., Sinha, A., Hass, S.L., Colman, S.S., Kumar, R.N., Brod, M. and 
Rowland, C.R. (2004) Validation of a General Measure of Treatment Satisfaction, the Treatment Satisfaction Questionnaire for Medication (TSQM), Using a National Panel Study of Chronic Disease. Health and Quality of Life Outcomes, 2, Article No. 12. https://doi.org/10.1186/1477-7525-2-12

[35] Sidani, S., Epstein, D.R., Fox, M. and Collins, L. (2018) The Contribution of Participant, Treatment, and Outcome Factors to Treatment Satisfaction. Research in Nursing \& Health, 41, 572-582. https://doi.org/10.1002/nur.21909

\section{Abbreviations}

BoNT/A = botulinum neurotoxin type A;

CP: cerebral palsy;

FDA: food drug association;

HFS: hemifacial spasm;

$\mathrm{M}$ : mean;

$\mathrm{SD}=$ standard deviation . 